\title{
Estudio Descriptivo De La Autonomía Motivacional En El Alumnado De Educación Secundaria De La Provincia De Benguela (Angola)
}

\author{
José Francisco Ferreira, (MSc) \\ Escuela de Enseñanza Secundaria de Educación General, \\ Baía Farta, Benguela (Angola) \\ María Pilar Cáceres Reche, (PhD)
}

Facultad de Ciencias de la Educación (Universidad de Granada, España)

Arturo Fuentes Cabrera, (PhD)

Facultad de Educación, Economía y Tecnología

(Ceuta, Universidad de Granada, España)

doi: 10.19044/esj.2017.v13n7p95 URL:http://dx.doi.org/10.19044/esj.2017.v13n7p95

\begin{abstract}
The study of motivation has a very important role in understanding of school success. The teachers and the education of parents and tutors are the key elements that influence the motivational dynamics for student learning, which is a crucial aspect to self-regulate the learning process in a positive way. This research aims to analyze the influence of the school context on the development of the motivational autonomy of Secondary Education students in the municipality of Baía Farta, Benguela province, Angola. For this purpose, a quantitative descriptive methodology was developed through a Likert scale questionnaire administered to teachers and students. The total sample of the study consisted of 650 students ( $60.1 \%$ men and $39.9 \%$ women) and 72 teachers (54.2\% men and $45.8 \%$ women). The main results point to the commitment and active participation of students through motivational strategies that are implemented through the activities planned by the school. Creating a supportive environment for the student to be motivated requires the development of a role of the guiding teacher, guide and support in the teaching and learning process, as opposed to a traditional conception of teaching more closed and hierarchical.
\end{abstract}

Keywords: Secondary education, learning, motivation, educational autonomy (Unesco thesaurus) 


\section{Resumen}

El estudio de la motivación tiene un papel muy importante en la comprensión del éxito escolar. Los maestros y la educación de los padres y tutores son los elementos clave que influyen en la dinámica motivacional para el aprendizaje del alumnado, siendo éste un aspecto crucial para autorregular el proceso de aprendizaje de forma positiva. Esta investigación presenta como objetivo analizar la influencia del contexto escolar en el segundo ciclo de Educación Secundaria de la Educación General No 3002 , del municipio de Baía Farta, provincia de Benguela (Angola), respecto al desarrollo de la autonomía motivacional de sus estudiantes. Para ello, se desarrolló una metodología descriptiva y cuantitativa, a través de un cuestionario tipo escala Likert, administrado al profesorado y alumnado. La muestra total del estudio consistió en 650 estudiantes (60,1\% hombres y $39,9 \%$ mujeres) y 72 profesores (54,2\% mujeres y 45,8\% mujeres). Los principales resultados apuntan al compromiso y la participación activa de los estudiantes, gracias a las actividades programadas por la escuela, dependiendo principalmente de las estrategias de motivación que se implementan. La creación de un entorno propicio para que el estudiante acepte y esté motivado por la escuela requiere desarrollar un rol del docente más como orientador, guía y apoyo en el proceso de enseñanza y aprendizaje y no, como suele aún manifestarse una concepción rígida, tradicional de la enseñanza de forma cerrada y jerarquizada.

Palabras clave: Educación Secundaria, aprendizaje, autonomía motivacional

\section{Introduction}

La autonomía en las escuelas ha sido una preocupación creciente en la literatura y ha sido definida desde diferentes perspectivas económicas, cultural, social. Así pues, en el Diccionario de la Lengua Portuguesa (DLP, 2015: p.105), la autonomía se define como "el derecho a gobernarse por sus propias leyes; la libre determinación; independencia.” En este sentido, Afonso (2014: 12) define la autonomía como "la capacidad de cualquier organización / entidad, individual o colectiva, tiene que ser capaz de gobernar por sus propias leyes, para actuar, para guiar, para resolver sus problemas, sin tener que recurrir a otro, suponiendo un cierto grado de independencia”. En el campo de la psicología, el concepto de autonomía se refiere al ámbito del“yo,” lo que significa, controlar su comportamiento, la experiencia y la guía del comienzo y la dirección de la acción. Para Reeve (2006), la autonomía no puede ser vista como una obra de mera casualidad o el resultado de cualquier espontaneidad, no sólo como una característica de la persona. Más bien, debe ser estudiada como un proceso contextualizado. Dentro de la escuela, la autonomía debe ser conceptualizada teniendo en 
cuenta las características de las instituciones educativas (supervisores, profesores) y la forma en que se articulan con la familia. Para estos autores, el ser humano sin la autonomía motivacional, no necesariamente atiende a factores externos o ambientales, sino más bien a una falta de interés (intrínseco) por las tareas de aprendizaje que debe ser analizado y promovido con la ayuda de la escuela.

En cuanto a la formación de la autonomía, como uno de los requisitos esenciales para el ser humano, desde el punto de vista de Freire, se afirma que "nadie es autónomo y luego decide. La autonomía se basa en la experiencia de varios, numerosas decisiones que se están tomando” (1996: 45). Según este autor (1996: 23), la autonomía como “maduración del ser sobre sí mismo, es un proceso, que se está produciendo. No aparece en la fecha programada. Es en este sentido que una pedagogía de la autonomía tiene que estar centrada en experiencias estimulantes de decisión y responsabilidad, es decir, en la experiencia respetuosa de la libertad”.

Por lo tanto, la autonomía es el resultado de un proceso de formación y maduración de la autorregulación motivacional, basada en la elección y la maduración del proceso de toma de decisiones guiada por los objetivos personales, intereses y valores. En esta misma línea, incluir la autonomía en el proceso de aprendizaje aporta un valor añadido a la formación de los estudiantes que sirven como apoyo para evaluar si éstos tienen o no el suficiente interés por las tareas como para participar de manera voluntaria y responsable en las actividades escolares.

El profesor en el aula tiene la tarea de inculcar el estilo, la voluntad, el interés por el aprendizaje, como valores esenciales para el desarrollo de la motivación intrínseca / independiente del sujeto. En paralelo, se debe crear un clima emocional del aula para facilitar el aprendizaje y el rendimiento escolar.

Según Lemos (2010:193), “es importante desarrollar en los alumnos la capacidad de auto-motivar, sobre todo porque la escuela hoy tiene que preparar a los estudiantes para la vida independiente y productiva.” La ley básica del sistema educativo Angolano (Ley de Educación, 2001: 191), establece: “la formación de la autonomía motivacional en el proceso de aprendizaje, es uno de los valores básicos de los que depende el buen desempeño de los docentes, en particular, la forma en que se crea ambientes que estimulan los sentimientos de pertenencia e interés hacia el aprendizaje.

Para Reeve (2006), la formación de la autonomía requiere un ambiente que de libertad a los estudiantes con el fin de que puedan por su propia iniciativa organizar, crear, de acuerdo con sus expectativas y su nivel de desarrollo psicomotor. Este clima de motivación no significa permisividad o negligencia, donde los estudiantes hacen lo que quieren sino entornos en 
los que el comportamiento esté impulsado por la elección, volición, estimular la auto-expresión y el derecho a la libre determinación.

Este mismo autor en 2002, citado por Teixeira (2007), considera que ayudar en la formación de la autonomía de los estudiantes requiere el cumplimiento de los procedimientos didácticos-pedagógicos, tales como la mejora de la competencia cognitiva, motivaciones, intereses y capacidades de los alumnos.

También implica el uso de un registro lingüístico, que fomenta el espíritu de la creatividad y la confianza en sí mismo, no la imposición. Para alcanzar estos objetivos, las estrategias de aprendizaje deben estar centradas en el estudiante (Pocinho y Canavarro, 2009). En particular, debe (a) facilitar el procesamiento de la información, (b) la consecución de los objetivos subyacentes del aprendizaje, y (c) tomar un conjunto de procedimientos planificados que requieren la organización de los medios con el fin de lograr ciertos objetivos. Para estos autores los estudiantes que implementan estrategias cognitivas y motivacionales muestran un rendimiento positivo de sus actividades escolares en comparación a aquellos estudiantes que no adoptan sus estrategias. La aplicación de estas estrategias por parte del estudiante es el resultado de la acción de los principales agentes educativos, que permiten al alumnado tomar decisiones propias, de forma responsable.

Partiendo de que la familia y la escuela son los contextos sociales que contribuyen al desarrollo y la educación de los estudiantes es fundamental promover una buena relación entre estos dos sistemas; por lo tanto, la relación entre ambos debe ser abierta y participativa.

Según Kaloustian (2002), la familia es el primer agente de socialización, insustituible para garantizar la supervivencia y la protección integral de los niños y otros miembros de la misma, independientemente de su situación familiar o la forma en que está estructurada. Es en la familia donde el individuo tiene el primer contacto con el mundo, el idioma, primeros valores y rutinas externas. Es la familia la que proporciona la base emocional para el desarrollo y el bienestar de sus miembros. Gokhale (1980) añade que la familia no es sólo el comienzo de la cultura y la base de la sociedad del futuro, sino también el centro de la vida social. Por tanto, el éxito en la educación del niño depende, en gran medida, del apoyo que la familia introduce en la creatividad y su comportamiento madurativo como un adulto.

Así pues, la interacción entre el binomio escuela y familia, se convierte en la base sólida para la vida social de los niños y su integración en la sociedad. Sin embargo, de acuerdo con Deci y Ryan (2000), los resultados de algunos estudios señalan la importancia de la escuela al promover actividades y estrategias para apoyar la autonomía a los estudiantes, que les permitan estar más motivados intrínsecamente, mostrando un mayor interés y 
disposición a aceptar desafíos. Del mismo modo, en este estudio se observó cómo los padres o tutores que fomentan la autonomía en el alumnado, éstos tienen resultados más satisfactorios en su motivación e interés para aprender, que los que promueven el control de la conducta. Otro estudio más reciente desarrollado en las escuelas secundarias de Brasil, pone de manifiesto la importancia del medio ambiente en la motivación de los estudiantes, que revela que las actividades en el contexto de la escuela permiten un aumento de su motivación autónoma y por lo tanto un mejor rendimiento. Según Clemente (2013), esto demuestra que es posible intervenir pedagógicamente, para que los estudiantes adquieran un papel más activo en el proceso de aprendizaje, y para el caso de Angola, resulta de gran interés atender al contexto socioeconómico y cultural donde nos ubicamos, con el fin de mejorar la difícil realidad educativa del país por los escasos recursos, infraestructuras y ofertas formativas para la población (Bumba, Cáceres y Ágreda, 2017).

\section{Problema de investigación}

En base a los supuestos teóricos y las contribuciones referidas anteriormente, esta investigación se formula en base al problema siguiente: análisis de las percepciones de la comunidad educativa (dirección escolar, profesorado, personal no docente, estudiantes y padres) en relación con la autonomía motivacional de los estudiantes del segundo ciclo de Educación Secundaria de Educación General $N^{\circ}$ 3002, en el municipio de Bahía Farta (Angola).

\section{Objetivos de la investigación}

Como objetivo general se pretende: "Analizar la influencia del contexto escolar angolano, de Baía Farta en el desarrollo de la autonomía motivacional de sus estudiantes." específicos:

De este objetivo principal se derivan otra serie de objetivos

1. Describir las percepciones de la comunidad educativa en relación a ese contexto escolar y su influencia en la construcción y el desarrollo de la autonomía motivacional de los estudiantes.

2. Identificar las expectativas de los tutores respecto al desarrollo de actividades y estrategias autónomas y motivadoras en el alumnado.

3. Verificar si las acciones desarrolladas por la escuela son lo suficientemente eficaces para la construcción y el desarrollo del aprendizaje autónomo y motivador en el alumnado. 


\section{Diseño del estudio}

\section{Metodología e instrumento}

Este estudio fue desarrollado mediante una metodología descriptiva, basada en un diseño no experimental por método de encuesta, a través del cuestionario, por tanto, un estudio cuantitativo, que pretende aproximarnos de una forma directa y general al contexto escolar de Baía Farta.

El instrumento, por tanto, aplicado tanto para el profesorado como para el alumnado, con un proceso previo de adaptación a cada colectivo, ha sido el cuestionario, tipo escala Likert con 4 niveles de respuesta: 1= totalmente en desacuerdo, 2= en desacuerdo, 3= de acuerdo y 4= totalmente de acuerdo. Éste ha seguido un proceso de elaboración“ad hoc”, cumpliendo con las características psicométricas exigidas, esto es, se realizó una validación de contenido por juicio de expertos y se halló un índice de fiabilidad alto (alfa de cronbach 0,8). En cuanto a su estructura, el cuestionario atiende a diferentes dimensiones: percepciones del entorno escolar (clima y la participación del centro y las familias en los estudiantes), el estilo motivacional hacia el estudio, el entorno personal en la escuela, las estrategias de aprendizaje, los estilos de crianza y la autonomía motivacional.

\section{Población y muestra}

En el municipio angolano, Baía Farta, (provincia de Benguela), existe una única escuela, por lo se cuenta con su participación en el estudio, como población diana. Esto significa que hay escasas alternativas para los estudiantes a la hora de elegir diferentes cursos.

Las dificultades han sido numerosas para acceder al profesorado y alumnado, puesto que ha sido necesario solicitar una distintos permisos al gobierno local y otras instancias gubernamentales. Fue posible gracias al apoyo proporcionado por el consejo escolar, quien estableció una serie de criterios para acceder a la muestra: responsabilidad en la disciplina de coordinación, las materias en las que los estudiantes tuvieron mayor y menor aprovechamiento en la escuela. Respecto a los estudiantes, los criterios seguidos se centran en: la autorización y consentimiento firmado por los padres.

Participaron finalmente seis miembros de la junta (Director de la escuela, los directores adjuntos: pedagógicos $\mathrm{y}$ administrativos, tres coordinadores de las disciplinas), cinco miembros del comité de padres y tutores y el resto fue elegido mediante un muestreo aleatorio, donde tomamos 650 estudiantes $(60,1 \%$ género masculino, 39,9\% género femenino) y 72 profesores (54,2\% hombres, 45,8\% mujeres) miembros de la junta, maestros, estudiantes y miembros del comité de padres y tutores que 
ejercen funciones en el segundo ciclo de Educación Secundaria, nº3002 municipio Baia - Farta, durante el curso académico 2014-2016.

\section{Análisis de resultados}

Se realizó un análisis estadístico descriptivo (porcentajes, frecuencias), a través del programa SPSS v.21, de acuerdo con los objetivos fijados.

En las siguientes tablas se encuentran los estadísticos descriptivos utilizados para los ítems personales y la caracterización de los participantes.

\section{* Profesores}

Como se puede observar en la tabla de frecuencias y porcentajes respecto al género, la representación masculina es ligeramente superior, con $54,2 \%$ al género femenino de la muestra (45,8\%).

Tabla 1. Análisis del género de los profesores (Elaboración propia)

\begin{tabular}{|c|c|c|}
\hline & Frecuencia & Porcentaje \\
\hline Masculino & 39 & 54,2 \\
\hline Femenino & 33 & 45,8 \\
\hline Total & 72 & 100,0 \\
\hline
\end{tabular}

En lo que respecta a la edad, la muestra se encuentra dividida en siete grupos. Por un lado, 23,6\% de los encuestados tienen una edad que va de los 26 a los 30 años; 15,3\% se encuentra en el grupo de los 31 a los 35 años; $12,5 \%$ entre los 36 y 40 años. Por otro lado, un cuarto de la muestra tiene edad entre los 41 y 45, los menores de 25 años respresentan el 8,3\% y de 46 a 50 años , sólo el 5,6\%.

Tabla 2 - Análisis de las edades de los profesores (Elaboración propia)

\begin{tabular}{|c|c|c|}
\hline & Frecuencia & Porcentaje \\
\hline$<25$ Años & 6 & 8,3 \\
\hline 26-30 Años & 17 & 23,6 \\
\hline 31 a 35 Años & 11 & 15,3 \\
\hline 36 a 40 Años & 9 & 12,5 \\
\hline 41 a 45 Años & 18 & 25,0 \\
\hline 41 a 45 Años & 7 & 9,7 \\
\hline 46 a 50 Años & 4 & 5,6 \\
\hline Total & 72 & 100,0 \\
\hline
\end{tabular}

En cuanto al tiempo de servicio en la profesión docente, más de la mitad de la muestra, tienen entre 6 y 10 años de experiencia, mientras que los profesores con menos de once años de experiencia, representan un 40,3\%.

Tabla 3 - Tiempo de servicio en la profesión docente (Elaboración propia)

\begin{tabular}{|c|c|c|}
\hline & Frecuencia & Porcentaje \\
\hline 6 a 10 Años & 43 & 59,7 \\
\hline$>11$ Años & 29 & 40,3 \\
\hline Total & 72 & 100,0 \\
\hline
\end{tabular}


En relación con el ítem del desempeño laboral en esta escuela, el $37,5 \%$ de los encuestados están en la misma escuela en un período de 0 a 5 años; en cuanto al 45,8\% de 6 a 10 años y el 16,7\% de 11 a 15 años. Estos datos son ilustrativos, pues la mayoría de los profesores trabajan ya bastante en esta institución, lo que les capacita para adoptar estrategias adecuadas a las características del contexto escolar, analizando la creación de ambientes propicios y estimulantes para el aprendizaje.

Tabla 4 - Tiempo de desempeño laboral en esta escuela (Elaboración propia)

\begin{tabular}{|c|c|c|}
\hline & Frecuencia & Porcentaje \\
\hline 0 a 5 años & 27 & 37,5 \\
\hline 6 a 10 años & 33 & 45,8 \\
\hline 11 a 15 años & 12 & 16,7 \\
\hline Total & 72 & 100,0 \\
\hline
\end{tabular}

En formación académica, más de la mitad del profesorado posee una Licenciatura en Ciencias de la educación, seguidos del Bachillerato y apenas un 10\% han realizado alguna Maestría en Educación.

Tabla 5 - Formación académica y profesional (Elaboración propia)

\begin{tabular}{|c|c|c|}
\hline & Frecuencia & Porcentaje \\
\hline Bachillerato & 17 & 23,6 \\
\hline Licenciatura en Ciencias de la Educación & 48 & 66,7 \\
\hline Maestría en Ciencias de la Educación & 7 & 9,7 \\
\hline Total & 72 & 100,0 \\
\hline
\end{tabular}

Se observa que más de la mitad de los profesores (58,3\%), permiten a los estudiantes desarrollar y comprometerse con las actividades elegidas por ellos mismos.

El grado de autonomía de los estudiantes es muy importante al ser el eje del proceso de enseñanza-aprendizaje. Por otro lado, el profesorado también permite a los estudiantes desarrollar actividades relacionadas con sus intereses, en su gran mayoría, más del 70\%. Sin embargo, todavía queda un $30 \%$ de la muestra que promueve un proceso de enseñanza-aprendizaje jerárquico, poco flexible y centrado en los estándares e indicadores establecidos por las diferentes políticas educativas.

Por otra parte, en relación con el ítem centrado en la reflexión del alumnado sobre los motivos para la elección o rechazo de las actividades, casi el 80\% están a favor de realizar este tipo de prácticas.

Por último, con respecto a la resolución de tareas complejas que permitan a los estudiantes la autoevaluación, se manifiesta un acuerdo pleno con más del 90\% del profesorado. 
Tabla 6- Percepciones sobre el desarrollo de la autonomía (Elaboración propia)

\begin{tabular}{|c|c|c|c|c|c|c|c|c|}
\hline & \multicolumn{2}{|c|}{\begin{tabular}{|c} 
Permito que los \\
alumnos desarrollen \\
actividades elegidas \\
por si mismos y que \\
se comprometan \\
con ellas
\end{tabular}} & \multicolumn{2}{|c|}{$\begin{array}{l}\text { Permito que los } \\
\text { alumnos } \\
\text { reflexionen sobre } \\
\text { los motivos de su } \\
\text { participación o } \\
\text { rechazo de } \\
\text { actividades }\end{array}$} & \multicolumn{2}{|c|}{$\begin{array}{c}\text { Permito que } \\
\text { los alumnos } \\
\text { desarrollan } \\
\text { actividades } \\
\text { relacionadas } \\
\text { con sus } \\
\text { intereses } \\
\end{array}$} & \multicolumn{2}{|c|}{$\begin{array}{c}\text { A través de } \\
\text { resolver tareas } \\
\text { complejas, permito } \\
\text { a los alumnos } \\
\text { autoevaluarse }\end{array}$} \\
\hline & Frec. & $(\%)$ & Frec. & $(\%)$ & Frec. & $(\%)$ & Frec. & $(\%)$ \\
\hline $\begin{array}{c}\text { Totalmente en } \\
\text { desacuerdo }\end{array}$ & 6 & 8,3 & 11 & 15,3 & 9 & 12,5 & 0 & 0,0 \\
\hline En desacuerdo & 9 & 12,5 & 8 & 11,1 & 15 & 20,8 & 0 & 0,0 \\
\hline De acuerdo & 42 & 58,3 & 29 & 40,3 & 37 & 51,4 & 39 & 54,2 \\
\hline $\begin{array}{c}\text { Totalmente de } \\
\text { acuerdo }\end{array}$ & 15 & 20,8 & 24 & 33,3 & 11 & 15,3 & 33 & 45,8 \\
\hline Total & 72 & 100,0 & 72 & 100,0 & 72 & 100,0 & 100,0 & 100,0 \\
\hline
\end{tabular}

En la tabla 7, se observa la importancia que el profesorado en prácticamente su totalidad atribuye a establecer un ambiente de apoyo, que favorezca el desarrollo del alumnado en proyectos más desafiantes y complejos. Por otro lado, de igual forma, coinciden plenamente en la necesidad de promover interacciones de apoyo y ayuda (100\%). Por tanto, se pone de manifiesto que la mayor parte de los profesores establece en sus clases una metodología participativa y colaborativa, aprendizaje entre iguales y retroalimentación permanente.

Tabla 7 - Percepción sobre el desarrollo y la cooperación (Elaboración propia)

\begin{tabular}{|c|c|c|c|c|}
\cline { 2 - 5 } & \multicolumn{2}{|c|}{$\begin{array}{c}\text { Establezco un ambiente de apoyo, que } \\
\text { favorezca el futuro desarrollo de los } \\
\text { alumnos en proyectos mas desafiantes y } \\
\text { complejos }\end{array}$} & $\begin{array}{c}\text { Estimulo las interacciones de } \\
\text { apoyo, de cooperación y ayuda }\end{array}$ \\
\hline De acuerdo & Frec. & $\mathbf{( \% )}$ & Frec. & $\mathbf{( \% )}$ \\
\hline $\begin{array}{c}\text { Totalmente de } \\
\text { acuerdo }\end{array}$ & 25 & 65,3 & 59 & 81,9 \\
\hline Total & 72 & 34,7 & 13 & 18,1 \\
\hline
\end{tabular}

\section{- Alumnos}

Atendiendo a la edad, gran parte de la muestra tiene menos de 14 años; el 32,4\% de los participantes tienen edades comprendidas entre los 15 e 17 años; el 22,8\% está entre los 18 a 20 años; y por último, más de 21 años son muy pocos $(3,9 \%)$. Los resultados de la muestra manifiestan, que la mayoría de los estudiantes, poseen las edades de acuerdo con el perfil establecido por el órgano de tutela la enseñanza en Angola, concretamente, el Ministerio de Educación. Siendo así, podemos considerar que se muestra una escolarización acorde con los patrones de edad definidos. 
Tabla 8 - Análisis de la edad de los alumnos (Elaboración propia)

\begin{tabular}{|c|c|c|}
\hline & Frecuencia & Porcentaje \\
\hline$<14$ años & 266 & 41,0 \\
\hline $15-17$ años & 210 & 32,4 \\
\hline 18 a 20 años & 148 & 22,8 \\
\hline$>21$ años & 25 & 3,9 \\
\hline Total & 649 & 100,0 \\
\hline
\end{tabular}

Respecto al género, existe una mayoría masculina $(60,1 \%)$, frente al casi 31\% de mujeres.

Tabla 9- Análisis de género (Elaboración propia)

\begin{tabular}{|c|c|c|}
\hline & Frecuencia & Porcentaje \\
\hline Masculino & 390 & 60,1 \\
\hline Femenino & 259 & 39,9 \\
\hline Total & 649 & 100,0 \\
\hline
\end{tabular}

El curso en el que se encuentran los encuestados, la mayor representación se encuentra en el $10^{\circ}$ año, con el 43,9\% de la muestra; el $23,6 \%$ en $11^{\circ}$ año y el $32,5 \%$ para el $12^{\circ}$ año. Así como, se corresponde con el área de estudio, perteneciente a las Ciencias Humanas (32,7\%), las Ciencias Físicas y Biología (35,6\%) y Ciencias Económicas y Jurídicas $(31,7 \%)$.

Tabla 10- Curso (Elaboración propia)

\begin{tabular}{|c|c|c|}
\hline & Frecuencia & Porcentaje \\
\hline $10^{\circ}$ año & 285 & 43,9 \\
\hline $11^{\circ}$ año & 153 & 23,6 \\
\hline $12^{\circ}$ año & 211 & 32,5 \\
\hline Total & 649 & 100,0 \\
\hline
\end{tabular}

En relación con el ítem de si frecuentemente el alumno se siente competente para realizar las tareas o trabajos en la escuela, las percepciones de los alumnos son positivas, en base a su capacidad. Esto queda patente con más del $80 \%$ de los estudiantes que muestran su disconformidad con este aspecto, y apenas un $20 \%$ de la muestra se siente identificado con esta afirmación.

Tabla 11-Frecuentemente no me siento competente en tareas escolares (Elaboración propia)

\begin{tabular}{|c|c|c|}
\hline & Frecuencia & Porcentaje \\
\hline Discordo plenamente & 197 & 30,4 \\
\hline Discordo & 289 & 44,5 \\
\hline Concordo & 99 & 15,3 \\
\hline Concordo plenamente & 64 & 9,9 \\
\hline Total & 649 & 100,0 \\
\hline
\end{tabular}


En esta misma línea, las percepciones que los alumnos tienen sobre la posibilidad de expresar sus ideas y opiniones en el aula, los resultados son mayoritariamente positivos (alrededor de un 70\%). Sin embargo, un 30\% aproximadamente consideran que no tienen esa posibilidad de reflexionar y decidir. En este sentido, es preciso tener en cuenta la metodología utilizada por los profesores, donde existen factores que pueden influir como: la edad, motivación, experiencia profesional y la cultura heredada de una educación tradicional donde la figura del profesor aparece apenas asociada a la instrucción, transmisión del conocimiento.

Tabla 12 - Siento que tengo libertad para elegir las cosas que deseo hacer en las aulas

(Elaboración propia)

\begin{tabular}{|c|c|c|}
\hline & Frecuencia & Porcentaje \\
\hline Totalmente en desacuerdo & 101 & 15,6 \\
\hline En desacuerdo & 94 & 14,5 \\
\hline De acuerdo & 314 & 48,4 \\
\hline Totalmente de acuerdo & 140 & 21,6 \\
\hline Total & 649 & 100,0 \\
\hline
\end{tabular}

Más de la mitad del grupo de estudiantes señalan que saben cómo estudiar (54,8\%); sin embargo el 45,1\% manifiestan que no saben cómo hacerlo, por lo que el grupo se muestra dividido, aportando un dato muy relevante de cara a replantearse la metodología seguida y la necesidad de incorporar cambios y mejoras en la planificación didáctica.

Tabla 13 - En general, sé como debo estudiar (Elaboración propia)

\begin{tabular}{|c|c|c|}
\hline & Frecuencia & Porcentaje \\
\hline Totalmente en desacuerdo & 100 & 15,4 \\
\hline En desacuerdo & 193 & 29,7 \\
\hline De acuerdo & 224 & 34,5 \\
\hline Totalmente de acuerdo & 132 & 20,3 \\
\hline Total & 649 & 100,0 \\
\hline
\end{tabular}

Estudiar, procurando repetir de forma oral la información, conforme se va leyendo un texto, suele ser la estrategia más utilizada prácticamente por la totalidad del grupo (90,8\%).

Tabla 14 - Para estudiar procuro repetir oralmente las informaciones, a medida que voy leyendo el texto (Elaboración propia)

\begin{tabular}{|c|c|c|}
\hline & Frecuencia & Porcentaje \\
\hline Totalmente en desacuerdo & 33 & 5,1 \\
\hline En desacuerdo & 27 & 4,2 \\
\hline De acuerdo & 384 & 59,2 \\
\hline Totalmente de acuerdo & 205 & 31,6 \\
\hline Total & 649 & 100,0 \\
\hline
\end{tabular}


Sin embargo, los alumnos están mayoritariamente en desacuerdo con la afirmación de que, para estudiar, siguen sólo los ejemplos dados por el profesor para memorizarlos (83,5\%). Esto indica que la memoria es necesaria y se emplea para el estudio, pero de forma conjunta se hace uso de la comprensión y asimilación del conocimiento para integrarlo de forma significativa y transferirlo a la vida cotidiana.

Tabla 15 - Cuando estudio para los exámenes, utilizo solamente ejemplos dados por el profesor e intento memorizarlos (Elaboración propia)

\begin{tabular}{|c|c|c|}
\hline & Frecuencia & Porcentaje \\
\hline Totalmente en desacuerdo & 211 & 32,5 \\
\hline En desacuerdo & 331 & 51,0 \\
\hline De acuerdo & 67 & 10,3 \\
\hline Totalmente de acuerdo & 40 & 6,2 \\
\hline Total & 649 & 100,0 \\
\hline
\end{tabular}

El ítem recogido en la tabla 16, pretende ser una valoración global de la importancia e interés que el alumnado manifiesta hacia la escuela, cuál es su motivación. Así pues, se destaca en la mayoría de los estudiantes (71,4\%) un interés hacia la escuela, frente al 30\% restante que no muestran esta motivación hacia la institución educativa. Este aspecto es fundamental pues es la base para facilitar el aprendizaje y el desarrollo de su formación integral.

Tabla 16-En general, me siento motivado para ir a la escuela (Elaboración propia)

\begin{tabular}{|c|c|c|}
\hline & Frecuencia & Porcentaje \\
\hline Totalmente en desacuerdo & 97 & 14,9 \\
\hline En desacuerdo & 89 & 13,7 \\
\hline De acuerdo & 197 & 30,4 \\
\hline Totalmente de acuerdo & 266 & 41,0 \\
\hline Total & 649 & 100,0 \\
\hline
\end{tabular}

\section{Discusión y conclusiones}

El objetivo de este estudio fue analizar la influencia del contexto escolar en el segundo ciclo de Educación Secundaria de la educación general $\mathrm{N}^{\mathrm{o}}$ 3002, en el municipio de Baía Farta (provincia de Benguela, Angola) a través del desarrollo de la autonomía motivacional de sus estudiantes.

Se pretendía identificar los factores contextuales que influyen en el desarrollo de la autonomía de los estudiantes, especialmente en un entorno poco estudiado como es el caso de las escuelas de Angola, lo que supone un valor añadido para este estudio.

Los datos analizados en este estudio se refieren a una caracterización sociodemográfica, en cuanto a la edad de los estudiantes y profesores, el tiempo de servicio y las características habituales de las escuelas este contexto. Los temas de estudio de la autonomía motivacional 
en las escuelas han sido ampliamente discutido en el contexto académico (por ejemplo, Ng et al, 2010 ;. Teixeira et al, 2012), y tienen un apoyo especial a través de autores como Deci y Ryan, 1985. La literatura hace hincapié en la importancia de crear un entorno de apoyo a las necesidades psicológicas básicas, a través del desarrollo de la estructura, la elección y la interacción. El apoyo a estas necesidades se ha traducido en la creación de un mejor ambiente de aprendizaje, volviendo al éxito de los estudiantes y también por la calidad del servicio prestado por los profesores (Niemiec y Ryan, 2009; Sosic-Valic, Keis, Lau, Spitzer y Streb, 2015). En este sentido, la mayoría de los estudiantes, como se refleja en la tabla 12, percibe este apoyo a la autonomía, en relación con la posibilidad de elegir la actividad en clase. Sin embargo, se reconoce que el cuestionario empleado, no permite conocer lo que quieren decir con autonomía ni sus respectivas dimensiones.

Por otra parte, los estudiantes parecen mostrar altos niveles de competencia en la clase. Una vez más, esta dimensión ha sido ampliamente revisada y sería preciso incluir en estudios e investigaciones futuras otros instrumentos más de corte cualitativo, como la entrevista semiestructurada o el grupo de discusión para precisar las dimensiones que alberga cada constructo desde las percepciones del alumnado y el profesorado, puesto que el cuestionario al ser un instrumento cuya respuesta se circunscribe a afirmaciones cerradas, no permite esta posibilidad. En el estudio realizado por McRae (2012) se presentan evidencias de que, las percepciones acerca de las competencias de los estudiantes, se asocia con mejores resultados en la lectura, la comprensión y contribuir a una mayor auto-eficacia en estos estudiantes, sin embargo, se descubrieron algunas diferencias culturales y étnicas entre los estudiantes. Las mismas que no se mantuvieron entre estudiantes afroamericanos y europeos especialmente en la auto-eficacia (McRae, 2012).

Estos datos avalan la consideración de que el compromiso y la participación activa de los estudiantes en las actividades programadas por la escuela, depende principalmente de las estrategias de motivación que se implementan. La creación de un entorno propicio para que el estudiante acepte los requisitos de la escuela es extremadamente importante para la motivación de este. Teniendo en cuenta las cuestiones planteadas y en función de los objetivos, el estudio desarrollado muestra una convergencia positiva de estos resultados. No obstante, ha habido ciertas limitaciones y es que el estudio fue desarrollado en las clases de secundaria, las mismas que fueron diseñadas para la enseñanza primaria; aunque no ha sido un factor determinante en su implementación. De hecho, la escuela de secundaria de segundo ciclo de la educación general nº3002 del municipio de Baía Farta, es de hecho un resorte motriz de la construcción y el desarrollo de la autonomía motivacional del alumnado, que permite una mayor participación de los 
estudiantes a interactuar y saber cómo aprender con el fin de mejorar el proceso de enseñanza y aprendizaje. Esto se debe principalmente a las competencias del perfil de los docentes para adaptarse a los requisitos de este nivel, así como la correcta aplicación de las estrategias de motivación. Esto se demuestra por el hecho de que la mayoría de los estudiantes han demostrado su acuerdo con el entorno escolar en la resolución de actividades.

La escuela tiene que ver con la formación de la autonomía del alumno, centrando sus acciones orientadas a satisfacer las necesidades de los estudiantes, que es esencial para la construcción de un ambiente educativo potencialmente motivador; permite igualmente a la libre determinación en la acción y el pensamiento, la estimulación de los procesos de toma de decisiones que les guíe a la satisfacción de los intereses y la motivación intrínseca. En lo que corresponde a la satisfacción de las necesidades a nivel de competencias, la escuela debe proporcionar las directrices que pongan retos de aprendizaje en un nivel óptimo, es decir, donde el grado de dificultad y complejidad de la tarea está en función de los conocimientos personales de los estudiantes. Uno de los componentes esenciales en la formación integral del estudiante y que ha sido implementado por la escuela tiene que ver con la formación en valores, que permite al estudiante concienciarse y sensibilizarse hacia la importancia de aplicar los conocimientos en la mejora y beneficio de la sociedad desde la integración y preocupación por el medio circundante.

Teniendo en cuenta las aportaciones de este trabajo, y su importancia en el contexto angolano, que está bastante ausente en los estudios de clima escolar, también hay que destacar el hecho de que los instrumentos de recolección de datos se han creado atendiendo a las características específicas de este país en el ámbito escolar, lo que supone un primer paso, para realizar estudios futuros e implementar medidas educativas que favorezcan el desarrollo en el alumnado de un aprendizaje autónomo, guiado por una motivación intrínseca y significativa para un desarrollo holístico, tanto en lo personal como en lo profesional.

\section{References:}

1. Afonso, A. E. (2014). El papel del maestro en la (re) construcción de Currículo 1 de la educación secundaria en Angola.

2. Bumba, F., Cáceres, Ma . P. y Ágreda, M. (2017). "Estudio evaluativo de la escuela rural en la provincia de Cabinda, Angola”. En Revista de Investigación Educativa (CPU-e), Universidad Veracruzana, Xalapa, México. Pp. 5-26.

3. Clemente, L. (2013). Autodeterminación y Educación para la investigación: Elementos estructurales para la Promoción de la 
Autonomía en las clases de física. Tesis Doctoral en Educación Científica de Ciencia y Tecnología - Universidad de Santa Catarina. Brasil.

4. Diccionario de la Lengua Portuguesa Acuerdo Ortográfico [en línea]. Puerto: Porto Editora (2015). Disponible en Internet: http://www.infopedia.pt/dicionarios/lingua-portuguesa/escola (último acceso el 05/02/2017).

5. Deci, E. L., \& Ryan, R. M. (2000). The" what" and" why" of goal pursuits: Human needs and the self-determination of behavior. Psychological inquiry, 11(4), 227-268.

6. Ley reguladora del Sistema Educativo en Angola (2001). Luanda Editorial.

7. Freire, P. (1996). Pedagogia da autonomia: saberes necessários à prática educativa. 13. ed. Rio de Janeiro: Paz e Terra.

8. Gokhale, G. (1980). A família desaparecerá? In Revista Debates Sociais $\mathrm{n}^{0}$ 30, ano XVI. Rio de Janeiro, CBSSIS.

9. Kaloustian, S. M. (2002). Família Brasileira: a base de tudo (5 edição). São Paulo: Cortez.

10. McRae, A. (2012). Teacher competence support for reading in middle school. Tese de Doutoramento. Universidade de Mariland.

11. Niemiec, C., e Ryan, R. (2009). Autonomy, competence, and relatedness in the classroom. Applying self-determination theory to educational practice. Theory and Research in Education, (7)2, 133144.

12. Pocinho, M., \& Canavarro, J. M. (2009). Sucesso escolar e estratégias de compreensão e expressão verbal: como compreender melhor as matérias e as aulas? Lisboa: Pedago.

13. Reeve, J. (2006). Motivação e emoção (4a edição). Rio de Janeiro: Editora Ltc.

14. Sosic-Vasic, Z., Keis, O., Lau, M., Spitzer, M., e Streb (2015). The impact of motivation and teachers' autonomy support on children's executive functions. Frontiers of Psychology, 6, 146. 